Published in final edited form as:

Curr Opin Gastroenterol. 2015 September ; 31(5): 395-399. doi:10.1097/MOG.0000000000000195.

\title{
Inflammation in Acute and Chronic Pancreatitis
}

\author{
Aida Habtezion \\ Department of Medicine, Division of Gastroenterology and Hepatology, Stanford University \\ School of Medicine, Stanford, California, 94305
}

\section{Summary}

\begin{abstract}
Immune cell contribution to the pathogenesis of acute and chronic pancreatitis is gaining more appreciation and further understanding in immune signaling presents potential therapeutic targets that can alter disease progression.
\end{abstract}

\section{Keywords}

Acute pancreatitis; chronic pancreatitis; high mobility group box 1; toll like receptor; macrophages

\section{Introduction}

Acute pancreatitis (AP) is one the most common gastrointestinal disorder leading to hospital presentation ${ }^{1}$. Common etiologic factors in adults in the United States include alcohol and gallstones. Patients who present with recurrent pancreatic injury or acute pancreatitis are at high risk for developing chronic pancreatitis ${ }^{2,3}$. Chronic pancreatitis most commonly is associated with alcohol in adults and leads to chronic abdominal pain associated with pancreatic acinar cell loss and fibrosis, which eventually can advance to exocrine and endocrine insufficiency ${ }^{4,5}$. Although most pancreatic cancer patients have no history of chronic pancreatitis, chronic pancreatitis is a very well known risk factor for pancreatic cancer ${ }^{6}$.

Lack of access to human pancreatic tissue during acute and chronic pancreatitis has made it difficult to obtain full understanding of pathogenic immune mechanisms involved.

Nevertheless, animal model studies even with their limitations and the scarce human studies continue to provide tools to advance our understanding of disease pathogenesis. In this review, recent data from animal and when available from human studies are discussed with focus on immune activation and responses. A separate article is dedicated to autoimmune pancreatitis and is not covered here.

Correspondence to: Aida Habtezion, MD MSc., Division of Gastroenterology and Hepatology, Department of Medicine, Stanford University School of Medicine, 300 Pasteur Drive, Stanford, CA, 94305, aidah@ stanford.edu. 


\section{Inflammation in Acute Pancreatitis}

There have been several clinical studies that investigated circulating cytokines as predictors and markers of disease severity in acute pancreatitis ${ }^{7}$. Pancreatic acinar cells particularly during the earlier stages of acute pancreatitis, produce cytokines such as tumor necrosis factor (TNF) $a^{8,9}$. These findings were recently corroborated with access to pancreatic acinar cells originating from patients with acute and recurrent acute pancreatitis ${ }^{10}$. In addition, the pancreatic acinar cells from these patients expressed cytokines such as IL-6 and IL-10, and the chemokine monocyte chemoattractant protein-1 (MCP-1; CCL2) suggesting that the initial inflammatory responses and signals that recruit leukocytes originate in injured pancreatic acinar cells.

Neutrophils play an important and pathogenic role in the early phase of acute pancreatitis development as they appear to be the first responder cells recruited to the injury site and contribute to activation of trypsinogen and progression to severe $\mathrm{AP}^{11,12}$. A more recent small study of circulating leukocytes in patients with severe acute pancreatitis with multiorgan dysfunction found aberant signaling and increased neutrophil transmigration properties ${ }^{13}$, suggesting that neutrophils contribute not only to the early local pancreas events but also to the systemic and end organ damages. Interestingly, a recent study using experimental model of acute pancreatitis found neutrophils to be recruited to the lung quite early and at times similar to that in the pancreas ${ }^{14}$.

The pathophysiology for the extra-pancreatic, especially lung inflammatory responses remain elusive. However, an elegant recent report showed a pathologic role for IL-6 transsignaling in promoting the lung injury ${ }^{15}$. Limited cells, mainly hepatocytes and leukocytes express membrane bound IL-6 receptor (IL-6R) and IL-6 mediated activation of IL-6R leads to a classic activation ${ }^{16}$. However, in addition to the membrane bound IL-6R, there is a soluble IL-6R (sIL-6R) that can bind IL-6 targets multitude of cells that express the ubiquitous gp130 receptor chain and don't necessarily express membrane bound IL-6R ${ }^{17}$. Unlike many other soluble cytokine receptors which act as inhibitors, the sIL-6R bound to IL-6 activates target cells and leads to IL-6 trans-signaling that is distinct from the classic IL-6 activation ${ }^{16}$. Interestingly IL-6 deficient mice had increased pancreatic damage whereas the lung injury was attenuated, but activation of IL-6 trans-signaling worsened both pancreatic and lung injuries suggesting distinct roles for IL-6-classic and IL-6-transsignaling pathways in driving the local versus distant pathologies. The findings also pointed at a possible protective and homeostatic role for IL-6 classical activation in the pancreas, which will need further evaluation. Another notable finding was that the myeloid cells were the source for the IL-6 associated lung injury ${ }^{15}$. A soluble protein that blocks IL-6-transsignaling is currently under clinical trial in other inflammatory diseases 16,18 and may be a promising target for severe acute pancreatitis associated lung and multi-organ failure.

Tissue injury associated innate immune activation and rapid neutrophil infiltration is usually followed by macrophage recruitment as shown in multiple inflammatory disorders including acute pancreaittis ${ }^{14,19}$. Perhaps related to the latter recruitment and long lasting effects of macrophages relative to neutrophils, most recent of inflammation related research in acute pancreatitis has focused on macrophages and innate immune signaling pathways. 
Neutrophils and macrophages play a dual role in inflammation and stimulate repair to allow healing process following injury, however, when such intricate balance is not achieved ongoing inflammation with neutrophils releasing interferon (IFN) $\gamma$ can lead to recruitment of pro-inflammatory macrophages that impair pancreatic regeneration and promote dedifferentiation of pancreatic epithelium as shown in a cerulein model of acute pancreatitis with or without additional oncogenic stimuli ${ }^{19,20}$. In contrast, a more recent report found a positive role for macrophages in pancreatic regeneration that is dependent on temporally synchronized macrophage polarity ${ }^{21}$. Although different animal models were used in these studies, the findings highlight the functional heterogeneity and plasticity of macrophages during induction and disease progression.

The prognosis of patients with acute pancreatitis strongly correlates with the presence of organ failure and infected pancreatic necrosis ${ }^{22}$. Intestinal barrier impairment has been shown with alcohol intoxication ${ }^{23}$, and factors such as hypotension and $\mathrm{TNFa}^{24,25}$ have been implicated as major contributors. Microbial components such as lipopolysaccharide (LPS) are potent activators of the host innate immune system via pathogen-associated molecular pattern (PAMP) recognition receptors such as toll like receptors (TLRs). The role of TLR4 in acute pancreatitis was demonstrated previously, where TLR4 deficient mice were protected against local and distant damages 26,27 . More recent data also showed support for either blocking or inactivating TLR4 using lactate or carbonmonoxide in experimentally induced acute pancreatitis 28,29 .

Tissue injury also releases endogeonous substances referred as damage-associated molecular pattern (DAMP) molecules, which can serve as TLR ligands and are being appreciated as important mediators for the pathogenesis of acute pancreatitis. One such molecule is the high mobility group box 1 (HMGB1), which belongs to a protein family of non-histone nuclear proteins that maintain chromosomal structure and regulate DNA-associated activities ${ }^{30}$. Remarkably, in apoptotic cells HMGB1 binds tightly to chromatin remnants, but in necrotic cells HMGB1 is passively released and triggers inflammatory response ${ }^{31}$. An interesting study using pancreas specific HMGB1 knockout mouse models showed dual roles for the HMGB1 where endogenous pancreatic HMBG1 was protective, but extracellular HMGB1 increased severity of acute pancreatitis ${ }^{32}$. Extracellular HMGB1 can activate TLRs and is one mechanism by which DAMP molecules allow immune activation and sensing of necrotic cells ${ }^{33}, 34$. In support of the above animal studies, a few clinical studies have reported correlation between circulating HMGB1 levels and severity of acute pancreatitis ${ }^{35,36}$.

IL-33, a recently recognized member of IL-1 superfamily of cytokines has been coined as an "alarmin" due to its release during cell injury, and similar to HMGB1 displays dual role as an intracellular nuclear factor and extracellularly as a cytokine by binding to surface receptor ST2 37,38 . Soluble IL-33 binds the Toll-interleukin 1 (IL-1) receptor (TIR) domaincontaining receptor ST2 and is thought to initiate immune-regulatory effects as well as Th2 immune responses; moreover a possible counterbalancing activity against pro-inflammatory effects of IL-1a and HMBG1 has also been suggested for IL-33 ${ }^{39}$. This notion is supported by a recent report of IL-33 promoting regulatory function in the intestine ${ }^{40}$. Although there is limited knowledge with regards to IL-33 and acute pancreatitis, Lemmers group correlated 
soluble ST2 (the decoy receptor) levels in plasma of acute pancreatitis patients with parameters of severity ${ }^{41}$. In addition using ST2-deficient mice, the group showed a protective role for ST2 with a potential regulartory function on mast cell activation during acute pancreatitis. More recently two groups reported opposing role for IL-33 in acute pancreatitis. The first report found pro-inflammatory role for exogenously delivered recombinant IL-33 protein in naive or non-pancreatitis mice where increased activation of ERK and NF- $\kappa$ B p65 subunit in the pancreas was associated with increased IL-6 and CXCL2 release from acinar cells, and acute inflammation in the pancreas but not in the lung or the jejunum ${ }^{42}$. The group also reported increased pancreatic IL-33 associated with mast cell activation in a distal bile-pancreatic duct ligation rodent model of acute pancreatitis but there were no intervention studies in the pancreatitis model. The second report utilized Coxsackievirus B (CVB) infection model of pancreatitis and also found elevated pancreatic IL-33 post infection, however exogenous IL-33 administration conferred protection and reduced viral titers ${ }^{43}$. In this study, ST2 deficient mice had decreased number of mast cells and were highly susceptible to CVB-induced pancreatic damage and necrosis. Interestingly, adoptive transfer of wild-type mast cells or alternatively activated macrophages (M2s) post infection protected the ST2 deficient mice from CVB-induced pancreatitis despite no alteration in viral load, which may suggest that protective role of IL-33 signaling in this model to be mediated in part via the well-defined wound healing and reparative roles of M2s. This was also consistent with the authors' observation that the adoptive transfer of mast cells was associated with an increase in pancreas M2 and regulatory T cell markers. The opposing roles of IL-33 reported by these two groups in the pancreas may relate to differences in models studied and the complex dual functions of IL-33 ${ }^{44}$ that may be context dependent, an area that will require further investigation with simultaneous examination of the cytokine and its receptors (ST2 and soluble ST2) in patients and different models of acute pancreatitis.

\section{Inflammation in Chronic Pancreatitis}

As mentioned in the above recurrent acute pancreatitis patients have a very high risk of developing chronic pancreatitis, for this reason experimental models of chronic pancreatitis have relied on repetitive injury. Notable histologic features in chronic pancreatitis include acinar cell atrophy, chronic inflammation, distorted or blocked ducts and invariably pancreatic stellate cell (PSC) activation associated with pancreatic fibrosis 5,45 . Earlier animal and human studies showed $\mathrm{T}$ cells and macrophages to be the predominant immune cell infiltrates in chronic pancreatitis ${ }^{46-48}$. A role for T cells in chronic pancreatitis has been proposed ${ }^{49,50}$. A potential role for macrophages in chronic pancreatitis was also proposed based on histologic observation of their close proximity to $\mathrm{PSCs}^{51,52}$. More recently, a study using cerulein model of chronic pancreatitis and human primary PSC-macrophage cocultures defined a pathogenic role for alternatively activated macrophages (M2) and IL-4 receptor (IL-4R)a signaling (Xue et al Nat Commun Accepted). Unlike in acute pancreatitis where M1s predominate, pancreas from mouse and human chronic pancreatitis were infiltrated with M2s. In addition, both mouse and human PSCs were a source for IL-4Ra ligands and promoted M2 polarization. The M2s in turn were efficient at activating the PSCs, prompting a "feed-forward" process that pointed a critical role for macrophages in 
pancreatic fibrosis. Notably, pancreatic fibrosis was reduced in mice with myeloid specific IL-4Ra deletion or mice receiving pharmacologic inhibitor of IL-4Ra following established disease.

Furthermore, human and mouse PSC driven M2 polarization was also inhibited with pharmacologic IL-4Ra blockade. Thus interfering with M2 polarization by targeting IL-4Ra signalling offers a potential mechanism for limiting fibrogenesis in chronic pancreatitis.

Host-microbiome interaction has been a central research topic over the past few years in health and disease. However, research in microbiome and pancreatic disease is still at its infancy. Farrell and co-workers using the Human Oral Microbe Identification Microarray noted alteration in salivary microbiota in patients with pancreatic cancer and chronic pancreatitis as compared to healthy subjects ${ }^{53}$. Whether these microbial alterations are confined to the oral mucosa or extend to the intestinal flora and how they relate to pathophysiology in the pancreas remain to be clarified. More recently, Neurath's group presented an oral abstract at the Digestive Disease Week May 2014 meeting, where experimental pancreatitis induced via forced expression of IL-17A in the liver of C57BL/6 was microbiota dependent ${ }^{54}$. They reported that institution reared C57BL/6, unlike industry purchased C57BL/6, developed chronic pancreatitis following over-expression of the IL-17A. Interestingly in the same model, the industry purchased mice developed chronic pancreatitis only following oral gavage of feces derived from their institution derived mice suggesting that the microbiota contributed to the pathogenesis of the IL-17A induced chronic pancreatitis. Although very limited data is available, further investigations will be awaited eagerly as microbiome related research resources are becoming more widely available and fecal microbiota transplant is becoming a widely accepted therapeutic option in different gastrointestinal diseases ${ }^{55-57}$.

\section{Conclusion}

The recent reports highlight the dynamic nature of inflammatory responses associated with acute and chronic pancreatitis. Timing is key and better diagnostic tools and biomarkers correlative to local and distant inflammatory signals need to be developed in order to prevent and target disease progression. Immune cells are critical in pancreatitis and have the ability to sense microenvironment, and respond to danger signals derived from endogenous and exogenous molecules. Further in depth study of environmental cues and immune responses in human and animal models are likely to improve our understanding of disease pathogenesis and offer means to change the unfavorable outcomes associated with the natural course of the acute and chronic pancreatitis.

\section{Acknowledgments}

None

Financial support or sponsorship: This work was supported in part by the Robert Wood Johnson Foundation grant and the National Institutes of Health Grant DK092421. 


\section{References}

1. Peery AF, Dellon ES, Lund J, et al. Burden of gastrointestinal disease in the United States: 2012 update. Gastroenterology. 2012; 143:1179-87. e1-3. [PubMed: 22885331]

2. Nojgaard C, Becker U, Matzen P, et al. Progression from acute to chronic pancreatitis: prognostic factors, mortality, and natural course. Pancreas. 2011; 40:1195-200. [PubMed: 21926938]

3. Yadav D, O'Connell M, Papachristou GI. Natural history following the first attack of acute pancreatitis. Am J Gastroenterol. 2012; 107:1096-103. [PubMed: 22613906]

4. Braganza JM, Lee SH, McCloy RF, et al. Chronic pancreatitis. Lancet. 2011; 377:1184-97. [PubMed: 21397320]

5. Witt H, Apte MV, Keim V, et al. Chronic pancreatitis: challenges and advances in pathogenesis, genetics, diagnosis, and therapy. Gastroenterology. 2007; 132:1557-73. [PubMed: 17466744]

6. Zheng L, Xue J, Jaffee EM, et al. Role of immune cells and immune-based therapies in pancreatitis and pancreatic ductal adenocarcinoma. Gastroenterology. 2013; 144:1230-40. [PubMed: 23622132]

7. Mayer J, Rau B, Gansauge F, et al. Inflammatory mediators in human acute pancreatitis: clinical and pathophysiological implications. Gut. 2000; 47:546-52. [PubMed: 10986216]

8. Norman JG, Fink GW, Franz MG. Acute pancreatitis induces intrapancreatic tumor necrosis factor gene expression. Arch Surg. 1995; 130:966-70. [PubMed: 7661681]

9. Gukovskaya AS, Gukovsky I, Zaninovic V, et al. Pancreatic acinar cells produce, release, and respond to tumor necrosis factor-alpha. Role in regulating cell death and pancreatitis. J Clin Invest. 1997; 100:1853-62. [PubMed: 9312187]

10. Gu H, Werner J, Bergmann F, et al. Necro-inflammatory response of pancreatic acinar cells in the pathogenesis of acute alcoholic pancreatitis. Cell Death Dis. 2013; 4:e816. [PubMed: 24091659]

11. Gukovskaya AS, Vaquero E, Zaninovic V, et al. Neutrophils and NADPH oxidase mediate intrapancreatic trypsin activation in murine experimental acute pancreatitis. Gastroenterology. 2002; 122:974-84. [PubMed: 11910350]

12. Abdulla A, Awla D, Thorlacius H, et al. Role of neutrophils in the activation of trypsinogen in severe acute pancreatitis. J Leukoc Biol. 2011; 90:975-82. [PubMed: 21810937]

13. Oiva J, Mustonen H, Kylanpaa ML, et al. Patients with acute pancreatitis complicated by organ dysfunction show abnormal peripheral blood polymorphonuclear leukocyte signaling. Pancreatology. 2013; 13:118-24. [PubMed: 23561969]

14**. Montecucco F, Mach F, Lenglet S, et al. Treatment with Evasin-3 abrogates neutrophil-mediated inflammation in mouse acute pancreatitis. Eur J Clin Invest. 2014; 44:940-50. This report describes the timely regulated leukocyte infiltration of the pancreas and lung during acute pancreatitis. [PubMed: 25132144]

15. Zhang H, Neuhofer P, Song L, et al. IL-6 trans-signaling promotes pancreatitis-associated lung injury and lethality. J Clin Invest. 2013; 123:1019-31. [PubMed: 23426178]

16*. Scheller J, Garbers C, Rose-John S. Interleukin-6: from basic biology to selective blockade of pro-inflammatory activities. Semin Immunol. 2014; 26:2-12. This report reviews IL-6 biology and the role of IL- 6 trans-signaling in progression of inflammatory diseases. [PubMed: 24325804]

17. Garbers C, Scheller J. Interleukin-6 and interleukin-11: same same but different. Biol Chem. 2013; 394:1145-61. [PubMed: 23740659]

18. Jones SA, Scheller J, Rose-John S. Therapeutic strategies for the clinical blockade of IL-6/gp130 signaling. J Clin Invest. 2011; 121:3375-83. [PubMed: 21881215]

19**. Folias AE, Penaranda C, Su AL, et al. Aberrant innate immune activation following tissue injury impairs pancreatic regeneration. PLoS One. 2014; 9:e102125. This report describes the role of innate immune activation in resolving inflammation and regeneration of the pancreas following injury. [PubMed: 25010227]

20. Liou GY, Doppler H, Necela B, et al. Macrophage-secreted cytokines drive pancreatic acinar-toductal metaplasia through NF-kappaB and MMPs. J Cell Biol. 2013; 202:563-77. [PubMed: 23918941] 
21**. Criscimanna A, Coudriet GM, Gittes GK, et al. Activated macrophages create lineage-specific microenvironments for pancreatic acinar- and beta-cell regeneration in mice. Gastroenterology. 2014; 147:1106-18. e11. This report describes how macrophage signaling and polarization affects and is required for pancreatic regeneration. [PubMed: 25128759]

22. Petrov MS, Shanbhag S, Chakraborty M, et al. Organ failure and infection of pancreatic necrosis as determinants of mortality in patients with acute pancreatitis. Gastroenterology. 2010; 139:813-20. [PubMed: 20540942]

23. Choudhry MA, Rana SN, Kavanaugh MJ, et al. Impaired intestinal immunity and barrier function: a cause for enhanced bacterial translocation in alcohol intoxication and burn injury. Alcohol. 2004; 33:199-208. [PubMed: 15596088]

24*. Thandassery RB, Yadav TD, Dutta U, et al. Hypotension in the first week of acute pancreatitis and APACHE II score predict development of infected pancreatic necrosis. Dig Dis Sci. 2015; 60:537-42. This study finds significant correlation hypotension and APACHE II score early during admission with acute pancreatitis with developing infected pancreatic necrosis. [PubMed: 24623313]

25*. Guo ZZ, Wang P, Yi ZH, et al. The crosstalk between gut inflammation and gastrointestinal disorders during acute pancreatitis. Curr Pharm Des. 2014; 20:1051-62. This report reviews factors associated with intestinal barrier disruption and intestinal inflammation during acute pancreatitis. [PubMed: 23782148]

26. Sharif R, Dawra R, Wasiluk K, et al. Impact of toll-like receptor 4 on the severity of acute pancreatitis and pancreatitis-associated lung injury in mice. Gut. 2009; 58:813-9. [PubMed: 19201771]

27. Awla D, Abdulla A, Regner S, et al. TLR4 but not TLR2 regulates inflammation and tissue damage in acute pancreatitis induced by retrograde infusion of taurocholate. Inflamm Res. 2011; 60:1093-8. [PubMed: 21863370]

$28 * *$. Hoque R, Farooq A, Ghani A, et al. Lactate reduces liver and pancreatic injury in Toll-like receptor- and inflammasome-mediated inflammation via GPR81-mediated suppression of innate immunity. Gastroenterology. 2014; 146:1763-74. This report describes immunoregulatory function of lactate via GPR81 in reducing liver and pancreatic injury. [PubMed: 24657625]

$29 * *$. Xue J, Habtezion A. Carbon monoxide-based therapy ameliorates acute pancreatitis via TLR4 inhibition. J Clin Invest. 2014; 124:437-47. This report describes protective role of carbonmonoxide and carbonmonoxide primed cells in protecting against acute pancreatitis mediated via TLR4 inactivation. [PubMed: 24334457]

30. Agresti A, Bianchi ME. HMGB proteins and gene expression. Curr Opin Genet Dev. 2003; 13:170-8. [PubMed: 12672494]

31. Scaffidi P, Misteli T, Bianchi ME. Release of chromatin protein HMGB1 by necrotic cells triggers inflammation. Nature. 2002; 418:191-5. [PubMed: 12110890]

32**. Kang R, Zhang Q, Hou W, et al. Intracellular Hmgb1 inhibits inflammatory nucleosome release and limits acute pancreatitis in mice. Gastroenterology. 2014; 146:1097-107. This report describes protective and regulatory role for intracellular pancreatic HMGB1 in acute pancreatitis. [PubMed: 24361123]

33. Miyake Y, Yamasaki S. Sensing necrotic cells. Adv Exp Med Biol. 2012; 738:144-52. [PubMed: 22399378]

34. Tian J, Avalos AM, Mao SY, et al. Toll-like receptor 9-dependent activation by DNA-containing immune complexes is mediated by HMGB1 and RAGE. Nat Immunol. 2007; 8:487-96. [PubMed: 17417641]

35. Yasuda T, Ueda T, Takeyama Y, et al. Significant increase of serum high-mobility group box chromosomal protein 1 levels in patients with severe acute pancreatitis. Pancreas. 2006; 33:359 63. [PubMed: 17079940]

36. Kocsis AK, Szabolcs A, Hofner P, et al. Plasma concentrations of high-mobility group box protein 1 , soluble receptor for advanced glycation end-products and circulating DNA in patients with acute pancreatitis. Pancreatology. 2009; 9:383-91. [PubMed: 19451748] 
37. Carriere V, Roussel L, Ortega N, et al. IL-33, the IL-1-like cytokine ligand for ST2 receptor, is a chromatin-associated nuclear factor in vivo. Proc Natl Acad Sci U S A. 2007; 104:282-7. [PubMed: 17185418]

38. Miller AM. Role of IL-33 in inflammation and disease. J Inflamm (Lond). 2011; 8:22. [PubMed: 21871091]

39. Gadina M, Jefferies CA. IL-33: a sheep in wolf's clothing? Sci STKE. 2007; 2007:pe31. [PubMed: 17565120]

40**. Schiering C, Krausgruber T, Chomka A, et al. The alarmin IL-33 promotes regulatory T-cell function in the intestine. Nature. 2014; 513:564-8. This report describes preferential expression of the IL-33 receptor ST2 in colon regulatory T cells where it supports regulatory function. [PubMed: 25043027]

41. Ouziel R, Gustot T, Moreno C, et al. The ST2 pathway is involved in acute pancreatitis: a translational study in humans and mice. Am J Pathol. 2012; 180:2330-9. [PubMed: 22542450]

42. Kempuraj D, Twait EC, Williard DE, et al. The novel cytokine interleukin-33 activates acinar cell proinflammatory pathways and induces acute pancreatic inflammation in mice. PLoS One. 2013; 8:e56866. [PubMed: 23418608]

43. Sesti-Costa R, Silva GK, Proenca-Modena JL, et al. The IL-33/ST2 pathway controls coxsackievirus B5-induced experimental pancreatitis. J Immunol. 2013; 191:283-92. [PubMed: 23733876]

44. Haraldsen G, Balogh J, Pollheimer J, et al. Interleukin-33 - cytokine of dual function or novel alarmin? Trends Immunol. 2009; 30:227-33. [PubMed: 19359217]

45. Apte MV, Pirola RC, Wilson JS. Pancreatic stellate cells: a starring role in normal and diseased pancreas. Front Physiol. 2012; 3:344. [PubMed: 22973234]

46. Emmrich J, Weber I, Nausch M, et al. Immunohistochemical characterization of the pancreatic cellular infiltrate in normal pancreas, chronic pancreatitis and pancreatic carcinoma. Digestion. 1998; 59:192-8. [PubMed: 9643678]

47. Goecke H, Forssmann U, Uguccioni M, et al. Macrophages infiltrating the tissue in chronic pancreatitis express the chemokine receptor CCR5. Surgery. 2000; 128:806-14. [PubMed: 11056444]

48. Deng X, Wang L, Elm MS, et al. Chronic alcohol consumption accelerates fibrosis in response to cerulein-induced pancreatitis in rats. Am J Pathol. 2005; 166:93-106. [PubMed: 15632003]

49. Hunger RE, Mueller C, Z'Graggen K, et al. Cytotoxic cells are activated in cellular infiltrates of alcoholic chronic pancreatitis. Gastroenterology. 1997; 112:1656-63. [PubMed: 9136845]

50. Schmitz-Winnenthal H, Pietsch DH, Schimmack S, et al. Chronic pancreatitis is associated with disease-specific regulatory T-cell responses. Gastroenterology. 2010; 138:1178-88. [PubMed: 19931255]

51. Detlefsen S, Sipos B, Feyerabend B, et al. Fibrogenesis in alcoholic chronic pancreatitis: the role of tissue necrosis, macrophages, myofibroblasts and cytokines. Modern pathology : an official journal of the United States and Canadian Academy of Pathology, Inc. 2006; 19:1019-26.

52. Deng X, Wang L, Elm MS, et al. Chronic alcohol consumption accelerates fibrosis in response to cerulein-induced pancreatitis in rats. The American journal of pathology. 2005; 166:93-106. [PubMed: 15632003]

53. Farrell JJ, Zhang L, Zhou H, et al. Variations of oral microbiota are associated with pancreatic diseases including pancreatic cancer. Gut. 2012; 61:582-8. [PubMed: 21994333]

54*. Leppkes M, Nowecki S, Wirtz SJ, et al. Intestinal Microbiota Contributes to the Pathogenesis of IL-17A Induced Chronic Pancreatitis. Gastroenterology. 2014; 146:S-68. This abstract presented at the DDW2014 describes a role for microbiota in the pathogenesis of IL-17 induced chronic pancreatitis.

55. Khoruts A, Dicksved J, Jansson JK, et al. Changes in the composition of the human fecal microbiome after bacteriotherapy for recurrent Clostridium difficile-associated diarrhea. J Clin Gastroenterol. 2010; 44:354-60. [PubMed: 20048681]

56. Grehan MJ, Borody TJ, Leis SM, et al. Durable alteration of the colonic microbiota by the administration of donor fecal flora. J Clin Gastroenterol. 2010; 44:551-61. [PubMed: 20716985] 
57*. Moayyedi P, Surette MG, Kim PT, et al. Fecal Microbiota Transplantation Induces Remission in Patients with Active Ulcerative Colitis in a Randomized, Controlled Trial. Gastroenterology. 2015 This study reports beneficial effect of fecal microbiota transplantation in inflammatory bowel disease. 


\section{Purpose of Review}

This report reviews recent animal model and human studies associated with inflammatory responses in acute and chronic pancreatitis. 


\section{Recent findings}

Animal model and limited human acute and chronic pancreatitis studies unravel the dynamic nature of the inflammatory processes and the ability of the immune cells to sense danger and environmental signals. In acute pancreatitis, such molecules include pathogen-associated molecular pattern recognition receptors such as toll like receptors, and the more recently appreciated damage-associated molecular pattern molecules or "alarmin" high mobility group box 1 (HMGB1) and IL-33. In chronic pancreatitis, a recent understanding of a critical role for macrophage-pancreatic stellate cell interaction offers a potential targetable pathway that can alter fibrogenesis. Microbiome research in pancreatitis is a new field gaining interest but will require further investigation. 


\section{Key Points}

Acute and chronic pancreatitis are dynamic inflammatory processes.

Immune cells play a critical role in pancreatitis progression.

Endogenous and exogenous signals provide immune cells a mechanism via which they can sense danger and environmental cues. 